\title{
Use of stochastic optimization techniques for damage detection in complex nonlinear systems
}

\author{
R. Jafarkhani ${ }^{1}$ and S.F. Masri ${ }^{1}$ \\ ${ }^{1}$ Sonny Astani Department of Civil and Environmental Engineering, Viterbi School of Engineering, University of \\ Southern California, Los Angeles, CA 90089-2531, USA
}

\begin{abstract}
In this study, the performance of stochastic optimization techniques in the finite element model updating approach was investigated for damage detection in a quarter-scale two-span reinforced concrete bridge system which was tested experimentally at the University of Nevada, Reno. The damage sequence in the structure was induced by a range of progressively increasing excitations in the transverse direction of the specimen. Intermediate non-destructive white noise excitations and response measurements were used for system identification and damage detection purposes. It is shown that, when evaluated together with the strain gauge measurements and visual inspection results, the applied finite element model updating algorithm on this complex nonlinear system could accurately detect, localize, and quantify the damage in the tested bridge columns throughout the different phases of the experiment.
\end{abstract}

\section{Introduction}

\subsection{Motivation and technical challenges}

The finite element model updating method has been studied for many years as a major research area within the field of Structural Health Monitoring (SHM), responding to an increasing demand for evaluating the integrity of civil infrastructures. Of particular relevance to potential applications of finite element model updating approaches for SHM in conjunction with dispersed systems are issues dealing with minimization of a complex, nonlinear, non-convex, high-dimensional cost function with several local minima. The fact that stochastic optimization techniques, such as evolutionary algorithms, simulated annealing and other random search methods have shown promising performance in solving global optimization problems is one of the main drivers for the growing interest in the investigation and implementation of these methods for large-scale finite element model updating approaches.

\subsection{Scope}

The main goal of the exploratory study reported in this article is to investigate the performance of stochastic optimization techniques in finite element model updating approaches for damage detection purposes. This paper partly reports the results in a previous publication of the authors [1].

\section{Overview of identification approach}

\subsection{Subspace method for system identification}

Subspace algorithms have been shown to be computationally very efficient and robust, especially for large data sets and large-scale systems. The subspace state-space system identification algorithm (the N4SID module in the System Identification Toolbox of MATLAB $^{\circledR}$ ) was employed in this study.

\subsection{Formulation of cost function}

For the study reported herein, the cost function to be minimized in the model updating process is calculated by cumulatively summing over the first $n$ dominant modes of the structure. Each term is the summation of two weighted, normalized values which quantify the deviation of the analytical frequency and mode shape from the corresponding measured ones. From this definition, the cost function can be written as follows:

$J(\underline{\boldsymbol{\alpha}})=\sum_{i=1}^{n}\left\{W_{f_{i}}\left|\frac{f_{i}^{(e)}-f_{i}^{(a)}}{f_{i}^{(e)}}\right|^{2}+W_{\overrightarrow{\boldsymbol{\varphi}}_{i}}\left[1-\frac{\left|\overrightarrow{\boldsymbol{\varphi}}_{i}^{(e)} \vec{\varphi}_{i}^{(a)}\right|^{2}}{\left(\overrightarrow{\boldsymbol{\varphi}}_{i}^{(e)} \overrightarrow{\boldsymbol{\varphi}}_{i}^{(e)}\right)\left(\overrightarrow{\boldsymbol{\varphi}}_{i}^{(a)} \vec{\varphi}_{i}^{(a)}\right)}\right]\right\}$

where $\underline{\boldsymbol{\alpha}}$ is the set of input parameters to be identified, $f_{i}$ and $\overrightarrow{\boldsymbol{\varphi}}_{i}$ represent the natural frequency and the modeshape of the $\mathrm{i}^{\text {th }}$ mode, and $W_{i}$ denotes the corresponding weight. Superscripts (e) and (a) stand for experimental and analytical results, respectively. 


\subsection{Optimization algorithm}

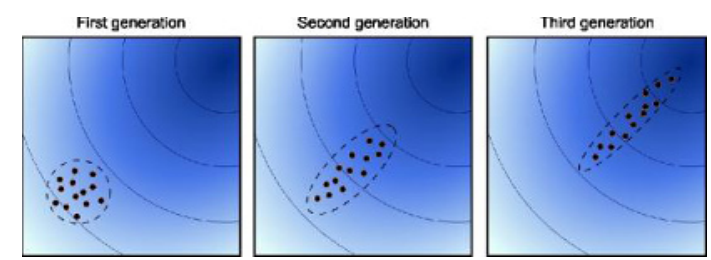

Fig. 1. Concept behind the covariance matrix adaptation for generation evolution for a 2-D optimization problem.

Two stochastic optimization techniques were investigated in this study. The first method is an Evolutionary Strategy based on Covariance Matrix Adaption (CMA-ES). CMAES was proposed for the first time in 1994 ([2]) and has been considerably developed since then. In this method, any new population is generated based on the multivariate normal mutation distribution with adapted covariance matrix. The adaption is based on increasing the likelihood of previously realized successful mutation steps, as well as exploiting the evolution path of the distribution mean of the strategy. Figure 1 schematically illustrates the main concept behind the covariance matrix adaptation for a 2-D optimization problem. As shown, the search direction is modified such that the candidate solutions in the new generation are more likely to be sampled along the principle axis.

The second optimization technique is a generic Genetic Algorithm (the GE module in the Optimization Toolbox of MATLAB®). Genetic Algorithms are based on a natural selection process that repeatedly modifies a population of individual solutions for an optimization problem through mimicking biological evolution.

\section{Experimental case study}

\subsection{Description of test bridge structure}

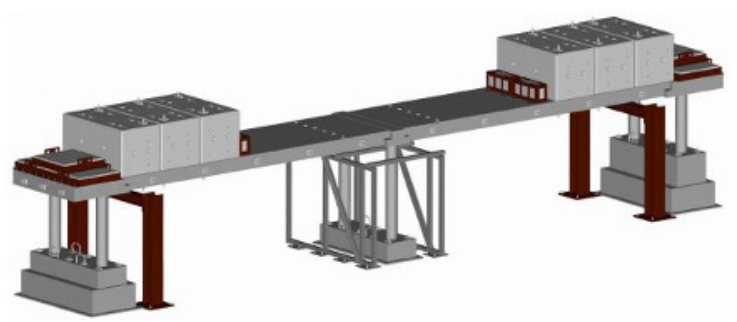

Fig. 2. Rendering of the bridge structure.

The case study to illustrate the application of the method under discussion is a two-span reinforced concrete bridge tested experimentally at the University of Nevada, Reno. The quarter-scale specimen has equal span-length of $30 \mathrm{ft}$ $(9.14 \mathrm{~m})$ and three double-column bents with variable column heights of $6 \mathrm{ft}(1.83 \mathrm{~m}), 8 \mathrm{ft}(2.44 \mathrm{~m})$, and $5 \mathrm{ft}$ $(1.52 \mathrm{~m})$, respectively. An amount of $280 \mathrm{kip}(1,245 \mathrm{kN})$ of additional mass is also provided on the slab to consider scaling effects. Figure 2 shows a rendering of the bridge structure.

\subsection{Destructive shaking procedure}

The bridge was tested under simulated excitations based on records from the Century City Country Club (1994 Northridge earthquake). Different levels of shaking amplitude, covering the range of PGA $=0.075 \times \mathrm{g} \sim 2.11 \times$ $\mathrm{g}$, were conducted on the structure. Intermediate, whitenoise excitations were also applied for system identification purposes ([3]).

\subsection{Instrumentation, data acquisition, and filtering}

Extensive instrumentation consisting of 298 channels was used to record the data at the frequency rate of $100 \mathrm{~Hz}$ ([3]). The following two scenarios were considered:

1) Only time-history records in both the longitudinal and transverse directions of the bridge obtained from the five accelerometers on the deck are available (4-D cost function).

2) Time-history records of the accelerometers on the deck as well as column curvature transducers are available (13-D cost function).

The first three transverse modes, in addition to the first longitudinal mode, were considered in the calculation of the cost function. The first transverse mode was identified as a dominant deck translation with slight in-plane rotation ( $\mathrm{f}=3.09 \mathrm{~Hz}$ ); the second mode as a dominant deck in-plane rotation with slight translation ( $f$ $=4.15 \mathrm{~Hz}$ ), and the third one as a deck bending ( $\mathrm{f}=12.90$ $\mathrm{Hz}$ ). The first longitudinal mode was in translation and dominated the longitudinal response $(f=2.99 \mathrm{~Hz}$ ). System identification results for the aforementioned modes with low-pass filtered data at $25 \mathrm{~Hz}$ or at any higher range were found to be identical. Consequently, a low-pass filter of $25 \mathrm{~Hz}$ was applied to all the processed records.

\subsection{NASTRN ${ }^{\circledR}$ finite element model}

With the assumption that the behavior of the tested bridge would stay in the linear region when it underwent low amplitude white-noise excitation, a NASTRAN ${ }^{\circledR}$ computer model was developed using linear beam column elements. Gross section properties were used for all of the elements, but the stiffness of the reinforced columns was calibrated and updated to represent the equivalent cracked moment of inertia. Finite element model updating for both scenarios was conducted to reproduce the response of the bridge specimen in the undamaged state. The identified parameters through the model updating process were modification factors to be applied to the gross section properties of the columns in the finite element model.

\subsection{Choice of initial parameters and weights}

Different sets of weighting factors in the model updating algorithm used in this study were evaluated for a synthetic damage detection scenario in the structure. 
Statistical data based on an ensemble of 100 simulations, with a maximum of 5,000 iterations for each test (needed due to the stochastic nature of the algorithm), were investigated to determine the proper selection of the identification parameters. For the weighting set of $\left\{\begin{array}{lll}4 & 2 & 1\end{array}\right.$ $1\})(4,2$, and 1 for the first three transverse modes, and 1 for the first longitudinal mode), the model updating algorithm was found to be capable of detecting, localizing, and quantifying the damage in all sections of the structure, with a high level of confidence $(95 \%$ level of confidence with an error margin of less than $4 \%$ for all parameters). Therefore, this weighting set was adopted in the rest of the study.

\section{Damage detection results}

\subsection{Preliminary damage detection}

A preliminary damage tracking technique was developed for this study using a recursive autoregressive moving average model (Recursive ARMA) to produce a near real-time (on-line) monitoring method to detect any significant change in the system response. The first-order single-output recursive ARMA structure to predict the value of the cost function is defined as

$$
(1+\emptyset L) J(t)=(1+\theta L) \epsilon(t)
$$

where $J$ is the real-time cost function value which is defined by Equation (1) based on extracted modal properties from a given window size of the past response record. $\varnothing$ and $\theta$ are autoregressive (AR) and moving average (MA) parameters, $\epsilon$ represents the white noise disturbance value and $L$ denotes the lag operator in time series analysis. Any change in the dynamic system throughout the experiment, resulting in the fluctuation of the value of the cost function, would be presumably reflected in the estimated parameters by the ARMA model. This procedure was implemented in MATLAB ${ }^{\circledR}$ (RARMAX module) for the response of the structure to the combined time history of the excitations in the transverse direction. A window size of $10 T_{1} \sim 30 T_{1}$, where $T_{1}$ is the fundamental period of the linearized system, for system identification, and a forgetting factor of $\lambda=0.95$ were found to be suitable for reliable instantaneous damage tracking of the investigated structure and therefore implemented in this study.

\subsection{Model updating results}

\subsubsection{Damage detection using input-output data}

The finite element model updating procedure was employed for both scenarios at different levels of damage to the structure. Figure 3 shows sample finite element model updating results using CMA-ES in scenarios 1 and 2 for white noise excitation records after damage induced in the structure due to a moderate earthquake (PGA = $0.18 \times \mathrm{g})$. Figure 4 illustrates the corresponding results obtained from GA optimization process. For scenario 1, (a) Scenario 1

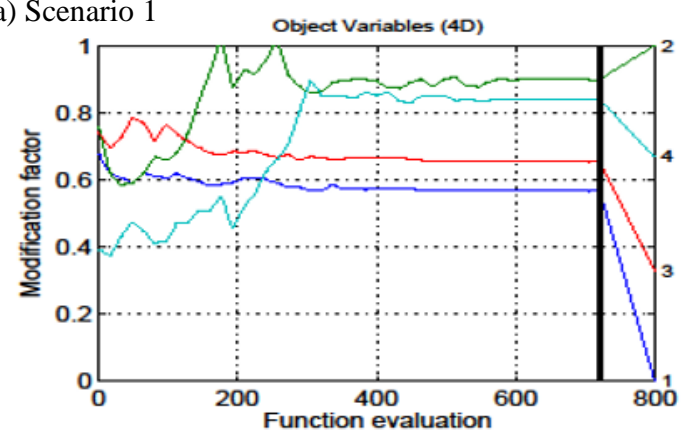

(b) Scenario 2

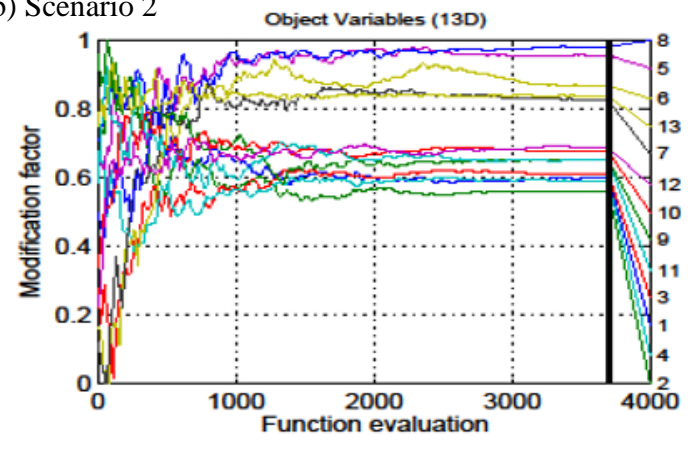

Fig. 3. Finite element model updating results of input-output data using CMA-ES

(a) Scenario 1

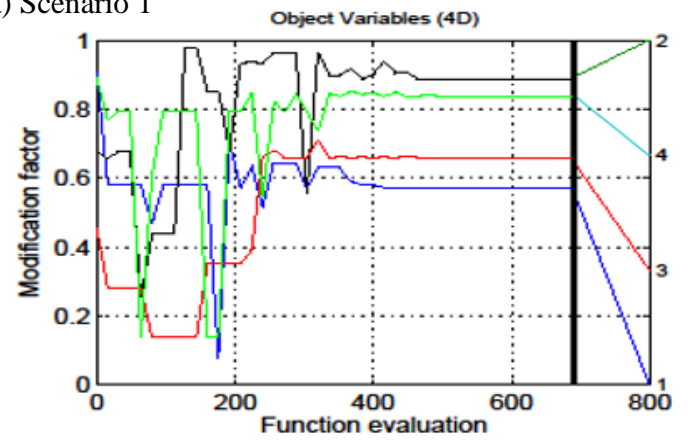

(b) Scenario 2

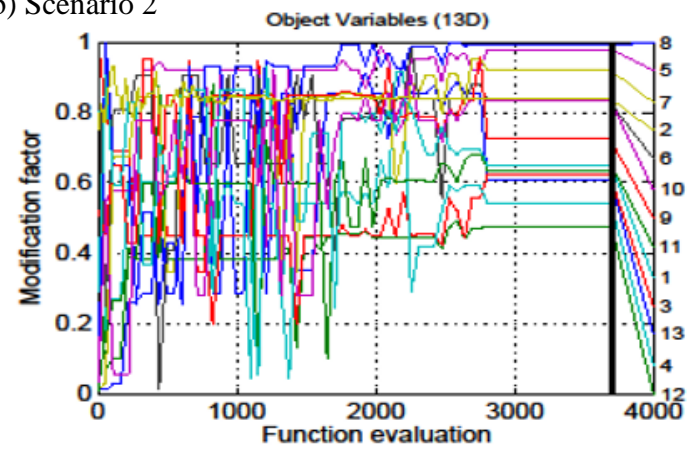

Fig. 4. Finite element model updating results of input-output data using GA

shown in figure $3 \mathrm{a}$ and figure $4 \mathrm{a}$, parameters 1-3 represent the remaining stiffness of the bents in the transverse direction and parameter 4 is the corresponding value for the longitudinal stiffness of all bents. As shown in figure $3 \mathrm{~b}$ and figure $4 \mathrm{~b}$ for scenario 2 , parameters 1-12 indicate the remaining stiffness in the transverse direction on the bottom and top of the columns and parameter 13 represents the corresponding value for the longitudinal stiffness of all bents. The variation of the modification 
factors with function evaluation through the optimization procedure are shown in each plot. Diminishing fluctuation of the parameters being identified through the optimization process indicates the convergence of these quantities to their final values.

\subsubsection{Damage detection using output-only data}

In continuous monitoring of a large-scale system, it is usually infeasible to excite the structure by a measurable artificial source, and even if possible, it will require expensive input devices such as shakers. Moreover, during real operation, the loading conditions may be substantially different from the ones used in the modal test. Therefore, there is a considerable tendency toward the use of freely available ambient excitation data sets for system identification and damage detection purposes. To evaluate the proposed approach for output-only data analysis, the damage detection procedures are conducted for both scenarios shown in figures 3 and 4 . As expected, comparison of the results with the corresponding quantities obtained from input-output data analysis shows an acceptable agreement, primarily due to the white-noise nature of the excitations.

\subsection{Validation results}

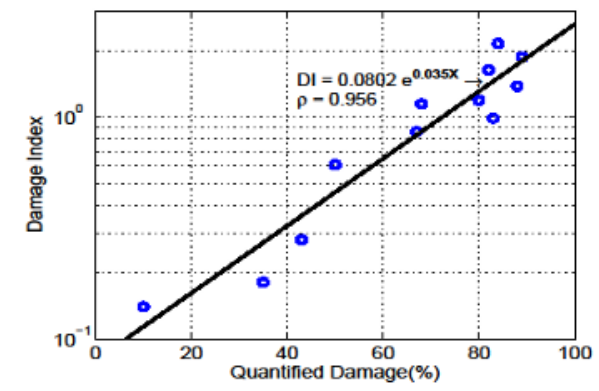

Fig. 5. Linear regression plot relating the NEES@Reno damage index and the quantified damage through model updating.

To quantitatively estimate the amount of damage to the structure, the NEES@Reno research team calculated a damage index for each bent and for each test motion using strain gauge records. This damage index, developed by Park and Ang ([4]) for reinforced concrete, is a practical measure of damage based on a combination of the amount of dissipated hysteretic energy, and the maximum displacement demand over ultimate displacement ratio. Values greater than 1.00 indicate collapse. However, the probability of collapse at this threshold is approximately $50 \%$ with a standard deviation of $\sigma=0.54$. Damage indices greater than 1.00 represent a higher probability of collapse ([3]).

Figure 5 illustrates the linear regression relating the damage index and the quantified damage through the model updating procedure. A (computed) correlation factor of $\rho=0.956$ strongly confirms the accuracy of the detected damage values qualitatively and quantitatively.

The damage-detection results of this article were also compared to the observations of the NEES@Reno team throughout the experiment. The investigation showed a fair agreement between the qualitative reported visual inspections and the quantitative identified damage indices through the model updating approach.

\section{Concluding remarks}

Based on the comparison of the results from the application of the finite element model updating algorithm under discussion with the strain gauge measurements and visual observations, the following conclusions can be made:

1) The simple ARMA model proposed for preliminary on-line damage detection can significantly increase the efficacy of the model updating process.

2) The finite element model updating algorithm used in this article was shown to be robust and accurate to detect, localize, and quantify the damage in synthetic simulations.

3) The finite element model updating algorithm presented and applied in this article could accurately detect and quantify the overall damage in the tested bridge bents throughout the experiment. Detected damage values are highly correlated $(\rho=0.956)$ with the damage index developed by Park and Ang ([4]), which is a practical measure of damage based on dissipated hysteretic energy and ductility demand.

4) The proposed method also showed very promising results for damage detection in the system using output-only data. This reveals the potentials of the technique to provide a useful tool for SHM purposes in conjunction with promising methods for identification of modal properties using freely available ambient vibration data.

Considering the promising performance of the optimization methods under discussion in solving wellknown benchmark problems of global optimization, the general conclusions from this study are useful in providing guidelines for the application of evolutionary strategies to real-world search problems, especially in the field of implementation of structural health monitoring for complex, nonlinear distributed systems.

\section{References}

1. Jafarkhani, R. \& Masri, S.F. (2011), Finite Element Model Updating Using Evolutionary Strategy for Damage Detection, Computer-Aided Civil and Infrastructure Engineering, 26(3), 207-224

2. Ostermeier, A., Gawelczyk, A. \& Hansen, N. (1994), A derandomized approach to self-adaptation of evolution strategies, Evolutionary Computation, 2(4), 369-380.

3. Johnson, N., Saiidi, M. \& Sanders, D. (2006), Largescale experimental and analytical studies of a twospan reinforced concrete bridge system, Technical Report CCEER-06-02, Center for Civil Engineering Earthquake Research, Department of Civil Engineering, University of Nevada, Reno, Nevada.

4. Park, Y. \& Ang, A. (1985), Mechanistic seismic damage model for reinforced concrete, Journal of Structural Engineering, 111(4), 722-739. 